\title{
Towards Spectral Efficiency Enhancement for loT Aided Smart Transportation: A Compressive OFDM Transmission and Regularized Recovery Approach
}

\section{Yusi Zhang}

Harbin Institute of Technology;Science and technology on Communication Networks Laboratory https://orcid.org/0000-0001-8525-0946

\section{Yong Li ( $\square$ young_li_54@126.com )}

Science and Technology on communication networks laboratory

\section{Xiaojie Fang}

Harbin Institute of Technology

\section{Xuejun Sha}

Harbin Institute of Technology

\section{Yuqing Feng}

Harbin Institute of Technology

Weizhi Wang

PengCheng laboratory

\section{Research Article}

Keywords: Bandlimited signal extrapolation, Compressed OFDM signal, Gerchberg-Papoulis algorithm, Regularization method, Spectral efficiency

Posted Date: November 8th, 2021

DOl: https://doi.org/10.21203/rs.3.rs-957524/v1

License: (c) (1) This work is licensed under a Creative Commons Attribution 4.0 International License. Read Full License

Version of Record: A version of this preprint was published at EURASIP Journal on Advances in Signal Processing on April 4th, 2022. See the published version at https://doi.org/10.1186/s13634-022-00843-w. 


\title{
Towards spectral efficiency enhancement for loT aided smart transportation: a compressive OFDM transmission and regularized recovery approach
}

\author{
Yusi Zhang ${ }^{1,2}$, Yong $\mathrm{Li}^{2 *}$, Xiaojie Fang ${ }^{1}$, Xuejun Sha ${ }^{1}$, Yuqing Feng ${ }^{1}$ and Weizhi Wang ${ }^{3}$
}

\author{
${ }^{*}$ Correspondence: \\ young_li_54@126.com \\ ${ }^{2}$ Key Laboratory of Science and \\ Technology on Communication \\ Networks, 050051 Shijiazhuang, \\ China \\ Full list of author information is \\ available at the end of the article
}

\begin{abstract}
The increasing number of vehicles brings ubiquitous connectivity and huge information interaction, implementing with limited spectrum resource. Focusing on the higher spectral efficiency requirement, a compressive OFDM system is proposed in this paper. The idea of compressing the transmission of OFDM signal for spectral efficiency enhancement origins from GP extrapolation algorithm for bandlimited signal. In the proposed scheme, a truncation filter with deliberately designed compressed ratio and truncation mode is performed on the OFDM signal to generate the compressive OFDM signal. At the receiver, up-sampling and iterative extrapolation are conducted to recover from the partial signal. Simulation results show that the compressive OFDM signal could be compressed up to 0.5 , presenting better compressive capability than the typical nonorthogonal SEFDM system. Further considering the ill-posed problem caused by the noise, a regularization approach is adopted to retain the convergence of recovery. Moreover, the proposed compressive OFDM system possesses the spectrally efficient advantage than SEFDM system. At the compressed ratio 0.5, the compressive OFDM system possesses better BER than SEFDM. At 10dB $E_{\mathrm{b}} / N_{0}$, the throughput rate of the compressive OFDM is 2 times and 1.6 times higher than OFDM and SEFDM, respectively.
\end{abstract}

Keywords: Bandlimited signal extrapolation; Compressed OFDM signal; Gerchberg-Papoulis algorithm; Regularization method; Spectral efficiency

\section{Introduction}

The Internet of Things (IoT) provides a medium to collaborate and correspond among numerous devices and sensors with applications and presents a broad application prospect for future wireless communication networks [1, 2]. The evolving Internet of Vehicle (IoV) networks is supposed to enable denser and rapider information interaction and connectivity to meet the requirements of mass services and applications for smart transportation system [3, 4, 5]. However, transmitting massive data within limited authorized spectral resources is one of the most urgent challenges $[6,7]$. Besides, inevitable spectrum conflict requires more effective spectrum management [8]. Therefore, technology with higher spectral efficiency is expected to relief the burden of spectrum scarcity and conflicts. Due to the wide application of orthogonal communication systems, a series of approaches focus on improving the spectral efficiency of Orthogonal Frequency Division Multiplexing (OFDM). It is known that the symbol rate of OFDM should not exceed the maximum rate of Nyquist criterion, otherwise the inter-symbol interference (ISI) would 
seriously affect the system performance. To fulfil the increasing spectral efficiency requirements, higher-order modulation methods are adopted. However, the sensitivity to noise and the impact of device nonlinear interference on the system will be larger with higher modulation order [9]. Moreover, several waveforms are proposed to lift the spectral efficiency for mass-data applications. Typically, multi-carrier technologies based on pulse shaping, such as Generalized Frequency Division Multiplexing (GFDM) [10], Universal-Filtered Multi-Carrier (UFMC) [11] and some efficient transmission methods based on Fast-Convolution Filter Bank (FCFB) [12], are proven effective in restraining the out-of-band radiation and inter-band interference. However, the spectrum efficiency within the signal bandwidth still remain unchanged in these existing schemes. In that case, research on more efficient transmission technique has always been concerned in IoT scenarios. Nonorthogonal transmission schemes are promising in achieving high spectral efficiency [13]. As for a system using a sinc function shaping filter, $25 \%$ more data can be transmitted than the orthogonal system within the same bandwidth [14]. According to the theoretical demonstration, Faster-Than-Nyquist method (FTN) is proposed for higher symbol transmission rate by adjusting the period of the shaping filter under the Nyquist period. Besides, a high spectral efficiency transmission method named spectrally efficient frequency division multiplexing (SEFDM) is proposed from the spectral compression aspect, also breaking through the Nyquist criterion in orthogonal systems [15]. These two non-orthogonal modulation techniques improve the spectrum efficiency at the cost of introducing ISI and inter-carrier interference, leading much detection difficulty [16]. It is proven that linear detection techniques like zero forcing detection perform undesirably. Thus, several nonlinear detection methods, such as iterative decoding (ID) and sphere decoding (SD), are conducted to retain the BER but with higher complexity $[17,18]$. Despite the higher spectrum efficiency of nonorthogonal methods, the improved effect was constrained by the Mazo limit which is upper-bounded at 0.8 with sinc shaping filter in frequency domain [19]. Extrapolation algorithms used to apply in medical imaging, optical signal processing and image reconstruction fields $[20,21,22]$. Due to the transmission or the detection environment, some segments of data are lost or seriously distorted. To complement the partial data, Gerchberg and Papoulis independently propose the GerchbergPapoulis extrapolation algorithm for band-limited continuous functions (referred as GP algorithm) [23, 24]. Later, the Sanz-Huang theorem for extrapolating discrete signals is provided, and the requirements of convergence for discrete GP extrapolation observation signals are discussed to evaluate the feasibility $[25,26]$. Also, the signal extrapolation methods are verified effective towards band-limited signals in higher-dimension or linear canonical transform(LCT) domain [27, 28]. Those analysis and conclusions indicate the applicability of extrapolation to recover the bandlimited signals.

The transmission scheme proposed in this paper is to realize high spectral efficiency communication, with the following properties and contributions:1. Based on the traditional OFDM transmission structure, the entire OFDM signal can be recovered from only half of it by GP algorithm. The compressed ratio as low as 0.5 will not generate self-interference on BER performance during extrapolation. 2. The recovery process is to extrapolate the orthogonal OFDM signal from non-orthogonal 
received compressive OFDM signal. A regularized extrapolator at the receiver is designed to restrain the influence of additive white noise, causing ill-posed extrapolation. To guarantee the accuracy of recovery, the actual extrapolating points should exceed the number of sub-carriers. 3. The proposed transmission scheme with simple linear decoder enables nearly $100 \%$ improvement on throughput rate compared to that of OFDM system with the same decoder and at least $60 \%$ improvement against SEFDM, but with higher computational complexity. The remaining of this paper is organized as follows. In Section 2, the GP algorithm is addressed as preliminary. In Section 3, compressive OFDM system model is given the error of the recovery algorithm and related compressed factors are designed to give out a recovery method. In Section 4, the simulation results of the BER and throughput rate of the system are presented to verify the improvements of the proposed method. Section 5 draws the conclusion of this paper.

\section{Preliminaries}

\subsection{GP algorithm}

The GP algorithm provides an iterative method to determine a bandlimited analytic function from a segment of it[23]. The premise of a feasible GP algorithm is that the original extrapolated $f(t)$ is bandlimited with finite energy.

$$
\begin{gathered}
F(\omega)=0,|\omega|>\Omega \\
\int_{-\infty}^{+\infty}|f(t)|^{2} d t=\int_{-\infty}^{+\infty}|F(\omega)|^{2} d \omega<+\infty
\end{gathered}
$$

where $F(\omega)$ denotes the Fourier transform result of $f(t)$, and $\Omega$ is a positive signal bandwidth.

Assume that only a finite segment $g(t)$ of $f(t)$ can be observed

$$
g(t)=f(t) p_{T}(t)
$$

where the operator $p_{T}$ truncates $f(t)$ within $[-T, T]$ and $\mathrm{T}$ is positive.

The process of the extrapolation follows Table 1, where Fourier Transform (FT) operator and the Inverse Fourier Transform (IFT) operator express as

$$
\mathcal{F}^{-1}\{X(\omega)\}=\int_{-\infty}^{\infty} X(\omega) e^{j \omega t} d t
$$

where $P_{\Omega}$ denotes a lowpass filter satisfying

$$
P_{\Omega}(\omega)=\left\{\begin{array}{l}
1,|\omega| \leq \Omega \\
0,|\omega|>\Omega
\end{array}\right.
$$

It can be found that each iteration consists of 4 main steps, named forward transform, filter, inverse transform, replacement. Note that the replacement process in Step 4 satisfies:

$$
g_{i}(t)=g(t)+\left(1-P_{T}(t)\right) f_{i}(t) .
$$


According to the Fourier transform (FT) pair of $\sin \Omega t / \pi t \leftrightarrow P_{\Omega}(\omega)$, the GP extrapolation process can be conducted as

$$
f_{i}(t)=f_{i-1}(t)+\int_{-T}^{T}\left(g(\tau)-f_{i-1}(\tau)\right) \frac{\sin \sigma(t-\tau)}{\pi(t-\tau)} d \tau .
$$

The GP algorithm is conducted under noiseless condition. Particularly, $f_{i}(t) \rightarrow$ $f(t)$ when $i \rightarrow \infty$ for any $t$. In the presence of noise, GP algorithm cannot theoretically guarantee convergence to the original function, nor can it obtain a convergent error.

Table 1 GP extrapolation for a bandlimited function.

\begin{tabular}{cc}
$\begin{array}{c}\text { Input } \\
\text { Initialization }\end{array}$ & $\begin{array}{c}\text { Finite segment } g(t), \text { maximum iterations ite; } \\
\text { The current iteration } i=1, g_{0}(t)=g(t)\end{array}$ \\
\hline Step1 & Do FT towards $g_{i-1}(t)$ and receive $G_{i-1}(\omega)=\mathcal{F}\left\{g_{i-1}(t)\right\} ;$ \\
Step2 & Filter the $G_{i-1}$ with $P_{\Omega}$ and receive $F_{i}(\Omega)=G_{i-1} \cdot P_{\Omega} ;$ \\
Step3 & Do IFT towards $F_{i}(\omega)$ and receive $f_{i}(t)=\mathcal{F}^{-1}\left\{F_{i}(\omega)\right\}$ \\
Step4 & $\begin{array}{c}\text { Exchange the segment of } f_{i}(t) \text { with the known segment } g(t) ; \\
\text { Step5 }\end{array}$ \\
$\begin{array}{c}\text { If } i \leq i t e, \text { the iteration proceeds from Step } 1 \text { and } i=i+1 ; \\
\text { Otherwise, output the result as } \hat{f}(t)=g_{i}(t) .\end{array}$ \\
\hline
\end{tabular}

\subsection{Expansion for bandlimited signal with prolate spheroidal functions}

Let $f(t)$ be $\Omega$-bandlimited, the expansion form with prolate spheroidal functions (PSF) can be expressed by

$$
f(t)=\sum_{k=0}^{\infty} \lambda_{k} \phi_{k}(t)
$$

where $\phi_{k}(t)$ denotes the $k$ th eigenfunction and the corresponding eigenvalue $\lambda_{k}$ is calculated from

$$
\lambda_{k}=\int_{-\infty}^{+\infty} f(t) \phi_{k}^{*}(t) d t
$$

The eigenfunction are totally orthogonal in the entire time domain and the interval $(-T, T)$,

$$
\begin{aligned}
& \int_{-\infty}^{+\infty} \phi_{k}(t) \phi_{l}^{*}(t) d t=\delta_{k, l}, \\
& \int_{-T}^{+T} \phi_{k}(t) \phi_{l}^{*}(t) d t=\lambda_{k} \delta_{k, l} .
\end{aligned}
$$

The eigenvalues are real, positive and ordered with descending value as

$$
1>\lambda_{0}>\cdots>\lambda_{k}>\cdots>0, \lim _{k \rightarrow \infty} \lambda_{k}=0
$$

The finite segment as shown in (2) can be expanded as

$$
g(t)=\sum_{k=0}^{\infty} \zeta_{k} \phi_{k}(t)
$$


where the eigenvalue is

$$
\zeta_{k}=\frac{1}{\lambda_{k}} \int_{-T}^{+T} g(t) \phi_{k}^{*}(t) d t
$$

It can be seen that the energy of $f(t)$ and $g(t)$ are both finite.

$$
\begin{aligned}
& \|f(t)\|^{2}=\sum_{k=0}^{\infty}\left|\lambda_{k}\right|^{2}<+\infty \\
& \|g(t)\|^{2}=\sum_{k=0}^{\infty}\left|\zeta_{k} \lambda_{k}\right|^{2}<+\infty
\end{aligned}
$$

\section{Methods}

This section introduces the main methods used in the compressive OFDM model. The generation method of compressive OFDM signal is proposed based on the bandlimited characteristic of traditional OFDM signal. Accordingly, the recovery method is given with analyzing the error considering influential parameters and noise.

\subsection{Generation method of compressive OFDM signal at transmitter}

An $N$-subcarrier OFDM signal with rectangular window function shaping filter can be expressed as

$$
s(t)=\frac{1}{\sqrt{T}} \sum_{n=0}^{N-1} x_{n} e^{\frac{j 2 \pi n t}{T}},
$$

where $T$ is the symbol period of OFDM signal and the subcarrier frequency space $\Delta f=1 / T$ satisfying the Nyquist orthogonality criterion. As the subcarrier waveform is the sum of a set of sinc functions, the bandwidth can be approximately calculated as $B_{O F D M}=N / T$. It is found that the OFDM signal can be regarded bandlimited with finite energy. According to the explanation of GP algorithm, the entire OFDM signal could be theoretically extrapolated from a segment of it. On this basis, the compressive OFDM system can be established as follows.

Figure 1 depicts the generation diagram of compressive OFDM signal based on the structure of traditional OFDM system. To obtain the compressive OFDM signal with compressed ratio $\alpha$, the traditional time-domain OFDM signal is processed by a truncation filter. In particular, the location of the reserved original OFDM signal is defined as the support set. The generation process is equivalent to the point product of the source and the truncation filter, defined as

$$
s^{\alpha}(t)=s(t) \cdot p_{\alpha}(t)
$$

where $s^{\alpha}(t)$ denotes the compressive OFDM signal, and $p_{\alpha}(t)$ is the truncation filter in the time domain satisfying

$$
p_{\alpha}(t)=\operatorname{rect}\left(\frac{t}{T}\right)=\left\{\begin{array}{l}
1, t \in T(\Psi), \\
0, t \notin T(\Psi),
\end{array}\right.
$$


where the rectangular window $\operatorname{rect}\left({ }^{\circ}\right)$ retains the data in the supporting set $\Psi$. The expression of the compression ratio is calculated by $\alpha=\sum T(\Psi) / T$.

Obviously, information beyond the supporting set in original OFDM is discarded via truncation filter. Whereas the truncated segment is relevant to the remaining segment (the compressed OFDM symbol) via DFT so that it still can be recovered. The relationship among the remaining segment, truncated segment and the original signal can be mathematically explained in Section 2.2. It is found that the entire bandlimited continuous OFDM signal and the truncated compressive OFDM signal can be expressed at the same prolate sphere basis. The former one can be determined by the latter one according to the analytical continuation principle [29]. In addition, the frequency spacing of compressive OFDM signals maintains while the symbol duration turns shorter by the compressed ratio $\alpha$, namely relationship of the symbol period of compressive OFDM and OFDM is $T_{c p s}=\alpha T$.

\subsection{Recovery method for compressive OFDM signal at receiver}

In Figure 2, the receiver structure can be illustrated on basis of OFDM system, with an extra extrapolator module correspondingly to recover the received signal.

The signal after $\mathrm{S} / \mathrm{P}$ should be up-sampled with the sample period $T_{\text {cps }} /(\alpha k N)$ , where $k$ represents the up-sampling rate. The up-sampling is required due to the constraint of recovery process based on GP algorithm. Take an $N$-point information source as an example, only $N / 2$-point signal is received and used to be extrapolated to obtain the following $N / 2$ points if the minimum Nyquist sampling rate is considered. As the description in Table 1 , the $N / 2$-point received compressive OFDM time domain signal is zero-tapped to $\mathrm{N}$ points at the end, and then undergoes $N$-DFT, lowpass filter, and $N$-IDFT. After that, the $N / 2$ points of the intermediate time domain result in the supporting set are replaced with those of received segment.

According to the description of the extrapolation filter, the lowpass filter retains all $N$ points and the points beyond the supporting set are still zero after IDFT. In that case, the above-mentioned iteration is invalid and no extra point is extrapolated. Therefore, up-sampling is necessary for the recovery for compressive OFDM signal. The influence of module parameters will be discussed in 3.3 .

\subsection{Parameter setting for compressive OFDM system}

\subsubsection{Compressed ratio and truncation filter at transmitter}

Note that the compressed ratio $\alpha$ and truncation filter $p_{\alpha}(t)$ should be designed in advance to determine a compressive OFDM signal. The mapping mode is BPSK and the bandwidth of extrapolation filter is the same as that of BPSK-OFDM signal. Three modes of truncation filter were compared as well, namely front segment, mid segment and back segment. Monte Carlo simulation on recovering compressive OFDM signals was carried out with different compressed ratios or truncation filters based on GP algorithm. The step size of compressed ratios was 0.01, and the maximum number of iterations was 100. The BER results were shown in Figure 3.

It can be seen from Figure 3 that the truncation filters remaining the front or back segment possesses comparable BER performance but better than that of mid truncation filter. For the front/back mode, the BER was relatively stable when the compressed ratio was not less than 0.50 when only considering the self-interference 
caused by the truncation process at the transmitter. Fluctuations of BER at $10^{-5}$ level were negligible when the compressed ratio ranged within 0.54-0.73. In that case, the minimum compressed ratio is appropriate to be implicated as 0.5 for compressive OFDM transmission scheme. It also implies a stronger resistance to self-interference in the generation process of compressive OFDM signals compared to SEFDM.

\subsubsection{Bandwidth of extrapolation filter}

As mentioned in Table 1, the bandwidth of the extrapolation filter is equal to that of the original function in terms of GP algorithm. With the increasing iterations, the error decreases and tends zero when the iterations tend infinite [25]. The rect function shaping filter is generally considered when analysing baseband OFDM signals, but the shaping filter could also be designed as Root Raised Cosine Filter (RRC) or other filters to improve the out-of-band radiation. Even so, the actual signal is not bandlimited strictly in the frequency domain. In terms of the recovery for the discrete signal, the length of the extrapolation filter reflects the filter bandwidth which is related to the points number of information source and upsampling rate. If the bandwidth of the extrapolation filter is selected as the cut-off bandwidth of the original OFDM symbol, possible aliasing error might be generated.

To evaluate the influence of the extrapolation filter bandwidth, the accuracy of extrapolating compressive OFDM with Rect shaping filter with different bandwidth is simulated, respectively. For BPSK-OFDM signal with 256 subcarriers, the compressed ratio is 0.5 and the received signal is up-sampled by 8 times before extrapolation. The up-sampled signal is recovered as revealed in Figure 2. Normalized mean square error (NMSE) is adopted to measure the accuracy performance, expressed as

$$
\mathrm{NMSE}=\frac{\|y-r\|^{2}}{\|r\|^{2}} .
$$

Defining the original signal points as $\omega$ which equals to the product of the number of subcarriers and up-sample times and the lowpass filter points as $\delta$, the NMSE values of the extrapolation by iteration filters with different bandwidth are illustrated in Figure 4.

As shown in Figure 4, iteration filter with the same bandwidth as that of the original signal obtains the best accuracy. The convergence of extrapolation turns slower if the filter is set with larger bandwidth, while the NMSE increases obviously with iterating when the filter is set with narrow bandwidth.

To explain with mathematical mechanism, the entire frequency domain signal could be divided into two parts as

$$
S(f)=L(f)+H(f)
$$

where $L(f)$ denotes the low-frequency signal at $|f| \leq \omega$, and $H(f)$ denotes the high-frequency signal at $|f|>\omega$. The corresponding time domain signal can be converted into

$$
s(t)=l(t)+h(t) .
$$


According to the linear additivity of the extrapolation, the result of $m$ th iteration can be expressed as

$$
r_{m}(t)=l_{m}(t)+h_{m}(t)
$$

The convergence of the $l_{m}(t)$ is determined by the bandlimited characteristics of $l(t)$ and the upper bound of the energy of $h_{m}(t)$ that could be inferred as

$$
E_{h_{m}}<m^{2} E_{h}
$$

where $E_{h_{m}}$ and $E_{h}$ denote the energy of $h_{m}(t)$ and $h(t)$, respectively. Note that the upper bound would increase with the number of iterations $m$ by the square times. However, the actual value is much smaller than the upper bound. When the out-of-band energy accounts for a small proportion of the total signal energy, the extrapolation error caused can be reduced [27], which might be attributed the considerable accuracy of $\delta / \omega=1$.

\subsubsection{Regularized extrapolator}

In addition to the errors caused by the extrapolation coefficients, the received signal also contains channel noise interference, which can be expressed as

$$
r(t)=s^{\alpha}(t)+z(t),
$$

where $s^{\alpha}$ represents the compressive OFDM signal with the compressed ratio 0.5 and the original symbol period T. $z(t)$ is Gaussian white noise with mean value 0 and variance $\delta^{2}$. The signal obtained in the $m$ th iteration can be described as

$$
r_{m}(t)=s_{m}^{\alpha}(t)+z_{m}(t)
$$

At that time, adopting the iterative filter with a bandwidth of $\delta / \omega=1$ and performing K-L expansion on $z(t)$ in the frequency domain, the extrapolated result of Gaussian distributed $z(t)$ in the first iteration can be obtained as

$$
z_{1}(t)=B \frac{\sin \delta t}{\pi t} \otimes \sum_{k=0}^{\infty} d_{k} \sqrt{\lambda_{k}} \phi_{k}(b t),
$$

where $B=\sqrt{\frac{\pi T}{\delta}}$ and $b=\frac{T}{2 \delta} ; \lambda_{k}$ and $\Phi_{k}$ are the eigenvalues and corresponding eigenfunctions of the K-L expansion. $d_{k}$ denotes the expansion coefficient, satisfying

$$
E\left\{d_{k} d_{r}^{*}\right\}=\left\{\begin{array}{cc}
S / \lambda_{k}, & k=r, \\
0, & k \neq r .
\end{array}\right.
$$

To simplify the convolution process of (25), the upper bound of the energy of $z_{1}(t)$ is calculated in the frequency domain as

$$
E\left\{\left|Z_{1}(f)\right|^{2}\right\}=2 S \pi b \sum_{k=0}^{\infty} \phi_{k}^{2}(b f)=2 S T
$$


where $S=\delta^{2} \Delta T$ and $\Delta T$ represents the sampling interval.

Due to the full-band Gaussian white noise, $Z m(f)$ still satisfies the Gaussian distribution. The mean square value of the energy of $Z m(f)$ can be expressed as

$$
\begin{aligned}
E\left\{\left|Z_{m}(f)\right|^{2}\right\} & =2 S \pi b \sum_{k=0}^{\infty}\left[\frac{1-\left(1-\lambda_{k}\right)^{n}}{\lambda_{k}}\right]^{2} \phi_{k}^{2}(b f) \\
& =2 S \pi b \sum_{k=0}^{\infty}\left(1+\varsigma_{k}+\cdots+\varsigma_{k}^{n-1}\right)^{2} \phi_{k}^{2}(b f),
\end{aligned}
$$

where $\varsigma_{k}=1-\lambda_{k}$ and $\lambda_{k} \in(0,1)$.

Inspired by [25], the upper bound of $E\left\{\left|Z_{m}(f)\right|^{2}\right\}$ can be inferred to $2 S T n^{2}$, similar to the result of (21). Nevertheless, the difference is that the noise is not correlated with the original signal and the actual impact of the extrapolating noise will accumulate with iterating. In that case, the extrapolation process turns into an ill-posed problem.

According to the GP algorithm, the extrapolation process can be expressed as

$$
\hat{S}=A \cdot S^{\alpha},
$$

where the operator $A$ contains the FT, filtering, IFT and exchange process. So is the recovery method for compressive OFDM expressed like that. Combining the idea of Landweber algorithm [30], the regularized recovery algorithm for compressive OFDM signal and the related receiver structure are shown in Figure 5.

In $m$ th iteration, the extrapolation calculation before replacement can be described as

$$
y_{m}^{\tau, \mu}=r_{m-1}+\mu\left(A^{*} 2 r-A^{*} A r_{m-1}-\tau r_{m-1}\right),
$$

where the regularized operator $A=F^{-1} \mathbf{H}_{\omega} F$, and $F$ as well as $F^{-1}$ are DFT matrix and IDFT matrix respectively. $\tau$ and $\mu$ are two parameters related to regularization, which are defined as regularized parameter and relaxation coefficient, respectively. When the extrapolator meets the judgement of iteration threshold, the iterative result $r_{m}$ would be output as the recovered $y$.

\section{Results and Discussion}

Table 2 provides key parameters at transmitter and receiver for compressive OFDM system, respectively. Corresponding variables are annotated in parentheses after the parameter names. The recovery is conducted on the premise of a synchronized receiver. As benchmark objects, the OFDM signal and SEFDM signal are selected both with 256 subcarriers. The generation of SEFDM signal with different compressed ratios could be referred to [15].

Table 2 Simulation parameter setting for compressive OFDM system

\begin{tabular}{cccc}
\hline Key parameters at transmitter & \multicolumn{2}{c}{ Key parameters at receiver } \\
\hline Subcarrier number & 256 & Extrapolation filter & $\delta / \omega=1$ \\
Compressed ratio $(\alpha)$ & $0.5-0.9$ & Maximum iteration & 100 \\
Truncated mode & Front & Regularized parameter $(\tau)$ & $10^{-5}, 10^{-6}, 10^{-7}$ \\
Up-sampled times & 8 & Relaxation coefficient $(\mu)$ & $5 \times 10^{-3}$ \\
\hline
\end{tabular}


Figure 6 illustrates the NMSE of recovering compressive OFDM signals with different compressed ratios from 0.5 to 0.9 . Without the interference of noise, the NMSE decreases with iterations, showing the convergence of the recovery. Besides, the recovery for signals with larger compressed ratios possesses faster convergence speed and lower NMSE. Obviously, more information could be reserved from truncation filter with larger support set. It is noted that the compressed OFDM signal is equivalent to the OFDM signal when the compressed ratio equals to 1. The NMSE reflects the accuracy of the recovery confirming the feasibility of the extrapolator.

To compare the compressive capability of compressive OFDM and SEFDM, Figure 7 presents the BER of compressive OFDM with compressed ratio 0.5 and SEFDM with compressed ratio 0.5-0.7 in AWGN channel. The iterative decoding (ID) is adopted in SEFDM system due to its non-orthogonality. The compressive OFDM signal is recovered with regularized extrapolator with $\tau=10^{-5}$. At compressed ratio 0.5-0.6, a significant BER performance degradation can be observed between compressive OFDM and SEFDM. Even adopting ID, the influence of ICI in SEFDM signals cannot be dismissed. It is attributed to the constraints of Mazo limit towards SEFDM system, namely the minimum Euclidean distance between symbol sequences reduced compared to orthogonal systems with the compressed ratio beyond Mazo limits [19]. In that case, the utilization of nonlinear detector (NLD) cannot compensate the degraded BER performance as usual. Furthermore, the performance of compressive OFDM with compressed ratio 0.5 and SEFDM with 0.7 is quite close to each other. When the $E_{\mathrm{b}} / N_{0}$ surpasses $4 \mathrm{~dB}$, the BER advantage of compressive OFDM tends bigger with less noise interference. In terms of the compressive capability, the superiority of compressive OFDM system is obvious against SEFDM.

Figure 8 compares the BER performance of the compressive OFDM, OFDM and SEFDM systems. The SEFDM with NLD adopts ID, while the SEFDM non NLD adopts traditional linear decision. In the compressive OFDM system, the receivers combined with regularized extrapolator (R-compressive OFDM, as shown in Figure 5) or not (compressive OFDM, as described in Section 3.2) all adopt linear decision. As can be seen, the SEFDM signal with 0.8 compressed ratio and NLD obtains approximate BER to that of OFDM, whereas the SEFDM with 0.8 compressed ratio but without NLD presents awful BER. The necessity of NLD towards SEFDM system can be inferred from the obvious BER difference. With the influence of noise, the BER of compressive OFDM without regularization only presents better than SEFDM with compressed ratio 0.5 and NLD. It verifies the Mazo limit on SEFDM again. Besides, the regularized iterations effectively improve the recovery result of compressive OFDM system. The convergence of extrapolator of compressive OFDM can be retained by regularized recovery method. Better robustness can be obtained with smaller regularized parameter $\tau$, but the improvement turns slight when $\tau<10^{-7}$. However, BER performance of compressive OFDM needs further improvements. In order to obtain BER at $10^{-4}$, the compressive OFDM system requires $1.6 \mathrm{~dB}$ and $1.2 \mathrm{~dB}$ higher of $E_{\mathrm{b}} / N_{0}$ than that of OFDM system and SEFDM system (0.8 compressed ratio, NLD), respectively. It can be found that the impact of noise can be restrained by regularized recovery method to some degrees, but cannot be dismissed. To solve this problem, design of regularized extrapolator for better BER performance will be conducted in the future research. 
Besides to the BER performance, the effective throughput rates (referred as ThroughputRate) of different systems are calculated to reflect the spectral efficiency in this paper. The ThroughputRate indicates the correct information could be transmitted per Hertz per second, defined as

$$
\text { ThroughputRate }=\frac{1-\text { BER }}{\alpha}
$$

As shown in Figure 9, the relationship of ThroughputRates of aforementioned systems satisfies that compressive OFDM $>$ SEFDM $>$ OFDM. Obviously, the difference in ThroughputRate is mainly attributed to the compressed ratio. SEFDM theoretically limits the compression ratio to not less than 0.8 with sinc funtion shaping [14]; the compressive OFDM system still has the ability to completely ignore self-interference when compressed to 0.5 and has better compression capabilities and systematic throughput rate. Under the same $E_{\mathrm{b}} / N_{0}$, the compressive OFDM system with regularized recovery method could obtain nearly 1.6 times and 2 times ThroughputRate compared to SEFDM system and OFDM system.

In terms of the computation complexity of compressive OFDM, the iteration threshold is set to be the maximum iterations M. From the (29), the complexity of the regularized recovery method is mainly determined by $M$ and the FFT points $N_{F F T}$, approximately equals to $O\left(M \cdot N_{\mathrm{FFT}} \cdot \log N_{\mathrm{FFT}}\right)$. It can be found that the computation increases linearly with $M$ and $N_{F F T}$, which can be controlled.

\section{Conclusions}

In this paper, a compressive OFDM system is proposed to deal with the scarcity of spectrum resource due to the requirement of massive information in the IoV aided smart transportation scenarios. The compressive OFDM signal is truncated from the front segment of original OFDM signal at the transmitter, and the compressed ratio can be as low as 0.5 . The recovery at the receiver is conducted with a upsampler and an extrapolator. To restrain the influence of additive white noise on the recovery, the regularization method is adopted with adjustable regularized parameter. Both theoretical deduction and simulation analysis are given to show the superiority of the proposed system. Results show that the compressive OFDM system possesses higher compressive capability, since the one with compressed ratio 0.5 performs better in BER than SEFDM with compressed ratio 0.5-0.7. Moreover, the compressive OFDM system outperforms in spectral efficiency performance. At the $10 \mathrm{~dB} E_{\mathrm{b}} / N_{0}$, the effective throughput rate of compressive OFDM is 2 times and 1.6 times than those of OFDM and SEFDM, respectively.

\footnotetext{
Abbreviations

loT:Internet of Things;loV:Internet of Vehicle;OFDM: Orthogonal Frequency Division Multiplexing;ISI: inter-symbol interference;GFDM:Generalized Frequency Division Multiplexing;UFMC:Universal-filtered multi-carrier;FCFB:Fast-convolution filter bank;FTN: Faster-than-nyquist;SEFDM: spectrally efficient frequency division multiplexing;ID:Iterative decoding;SD:Sphere decoding;GP algorithm:Gerchberg-Papoulis extrapolation algorithm; LCT:Linear canonical transform;FT:Fourier transform;IFT:Inverse Fourier transform.

Declarations

Ethics approval and consent to participate

No applicable.
} 
Consent for publication

The manuscript does not contain any individual person's data in any form (including individual details, images, or videos) and therefore the consent to publish is not applicable to this article.

Availability of data and materials

Not applicable.

Competing interests

The authors declare that they have no competing interests.

Funding

The research activities described in this paper have been conducted within the Natural Science Foundation of Heilongjiang Province of China under Grant YQ2021F003, National Natural Science Foundation of China under Grant 62171151 and Science and Technology on Communication Networks Laboratory under Grant SCX21641X003.

Author's contributions

$Y Z$ wrote and editted this manuscript. $Y Z$ and $X S$ conceptualized this study. $X F$ and $Y L$ refined the idea. All authors read and approved the final manuscript.

\section{Acknowledgements}

The authors would like to acknowledge all the participants for their contributions to this research study.

Author details

${ }^{1}$ School of Electronics and Information Engineering, Harbin Institute of Technology, Xidazhi Street, 150001 Harbin, China. ${ }^{2}$ Key Laboratory of Science and Technology on Communication Networks, 050051 Shijiazhuang, China.

${ }^{3}$ Peng Cheng Laboratory, 518101 Shenzhen, China.

\section{References}

1. Liu, C., Feng, W., Chen, Y., Wang, C.-X., Ge, N.: Cell-free satellite-uav networks for $6 g$ wide-area internet of things. IEEE Journal on Selected Areas in Communications 39(4), 1116-1131 (2021). doi:10.1109/JSAC.2020.3018837

2. Cheng, J., Yuan, G., Zhou, M., Gao, S., Liu, C., Duan, H., Zeng, Q.: Accessibility analysis and modeling for iov in an urban scene. IEEE Transactions on Vehicular Technology 69(4), 4246-4256 (2020). doi:10.1109/TVT.2020.2970553

3. Xu, L., Wang, H., Gulliver, T.A.: Outage probability performance analysis and prediction for mobile iov networks based on ics-bp neural network. IEEE Internet of Things Journal 8(5), 3524-3533 (2021). doi:10.1109/JIOT.2020.3023694

4. Liu, X., Hu, S., Li, M., Lai, B.: Energy-efficient resource allocation for cognitive industrial internet of things with wireless energy harvesting. IEEE Transactions on Industrial Informatics 17(8), 5668-5677 (2021). doi:10.1109/TII.2020.2997768

5. Contreras-Castillo, J., Zeadally, S., Guerrero-lbañez, J.A.: Internet of vehicles: Architecture, protocols, and security. IEEE Internet of Things Journal 5(5), 3701-3709 (2018). doi:10.1109/JIOT.2017.2690902

6. Liu, X., Zhang, X.: Rate and energy efficiency improvements for $5 \mathrm{~g}$-based iot with simultaneous transfer. IEEE Internet of Things Journal 6(4), 5971-5980 (2019). doi:10.1109/JIOT.2018.2863267

7. Akinsanya, A., Nair, M., Pan, Y., Wang, J.: A dynamic resource allocation scheme in vehicular communications. In: IEEE INFOCOM 2020 - IEEE Conference on Computer Communications Workshops (INFOCOM WKSHPS), pp. 127-131 (2020). doi:10.1109/INFOCOMWKSHPS50562.2020.9162889

8. Cai, P., Zhang, Y.: Intelligent cognitive spectrum collaboration: Convergence of spectrum sensing, spectrum access, and coding technology. Intelligent and Converged Networks 1(1), 79-98 (2020). doi:10.23919/ICN.2020.0006

9. Bang, H., Park, C.-S.: Analysis of modulation order to guarantee real-time service throughputs in ofdma-pon. IEEE Communications Letters 19(4), 549-552 (2015). doi:10.1109/LCOMM.2015.2401561

10. Michailow, N., Matthé, M., Gaspar, I.S., Caldevilla, A.N., Mendes, L.L., Festag, A., Fettweis, G.: Generalized frequency division multiplexing for 5 th generation cellular networks. IEEE Transactions on Communications 62(9), 3045-3061 (2014). doi:10.1109/TCOMM.2014.2345566

11. Chen, X., Wu, L., Zhang, Z., Dang, J., Wang, J.: Adaptive modulation and filter configuration in universal filtered multi-carrier systems. IEEE Transactions on Wireless Communications 17(3), 1869-1881 (2018). doi:10.1109/TWC.2017.2786231

12. Yli-Kaakinen, J., Levanen, T., Valkonen, S., Pajukoski, K., Pirskanen, J., Renfors, M., Valkama, M.: Efficient fast-convolution-based waveform processing for $5 \mathrm{~g}$ physical layer. IEEE Journal on Selected Areas in Communications 35(6), 1309-1326 (2017). doi:10.1109/JSAC.2017.2687358

13. Mazo, J.E.: Faster-than-nyquist signaling. The Bell System Technical Journal 54(8), 1451-1462 (1975). doi:10.1002/j.1538-7305.1975.tb02043.x

14. Wang, K., Liu, A., Liang, X., Peng, S., Zhang, Q.: A faster-than-nyquist (ftn)-based multicarrier system. IEEE Transactions on Vehicular Technology 68(1), 947-951 (2019). doi:10.1109/TVT.2018.2861823

15. Ma, Y., Wu, N., Yuan, W., Ng, D.W.K., Hanzo, L.: Joint channel estimation and equalization for index-modulated spectrally efficient frequency division multiplexing systems. IEEE Transactions on Communications 68(10), 6230-6244 (2020). doi:10.1109/TCOMM.2020.3007387

16. Anderson, J.B., Rusek, F., Öwall, V.: Faster-than-nyquist signaling. Proceedings of the IEEE 101(8), 1817-1830 (2013). doi:10.1109/JPROC.2012.2233451

17. Yuan, W., Wu, N., Guo, Q., Ng, D.W.K., Yuan, J., Hanzo, L.: Iterative joint channel estimation, user activity tracking, and data detection for ftn-noma systems supporting random access. IEEE Transactions on Communications 68(5), 2963-2977 (2020). doi:10.1109/TCOMM.2020.2975169 
18. Guo, M., Qiao, Y., Zhou, J., Tang, X., Qi, J., Liu, S., Xu, X., Lu, Y.: Ici cancellation based on mimo decoding for ftn non-orthogonal fdm systems. Journal of Lightwave Technology 37(3), 1045-1055 (2019). doi:10.1109/JLT.2018.2885791

19. Mazo, J.E., Landau, H.J.: On the minimum distance problem for faster-than-nyquist signaling. IEEE Transactions on Information Theory 34(6), 1420-1427 (1988). doi:10.1109/18.21281

20. Seiler, J., Kaup, A.: Complex-valued frequency selective extrapolation for fast image and video signal extrapolation. IEEE Signal Processing Letters 17(11), 949-952 (2010). doi:10.1109/LSP.2010.2078504

21. Fridolin, I., Karai, D., Kostin, S., Ubar, R.: Accurate dialysis dose evaluation and extrapolation algorithms during online optical dialysis monitoring. IEEE Transactions on Biomedical Engineering 60(5), 1371-1377 (2013). doi:10.1109/TBME.2012.2234458

22. Biondi, F.: Recovery of partially corrupted sar images by super-resolution based on spectrum extrapolation IEEE Geoscience and Remote Sensing Letters 14(2), 139-143 (2017). doi:10.1109/LGRS.2016.2615564

23. Papoulis, A.: A new algorithm in spectral analysis and band-limited extrapolation. IEEE Transactions on Circuits and Systems 22(9), 735-742 (1975). doi:10.1109/TCS.1975.1084118

24. Gerchberg, R.W.: Super-resolution through error energy reduction. Optica Acta: International Journal of Optics 21(9), 709-720 (1974). doi:10.1080/713818946

25. Sanz, J., Huang, T.: Some aspects of band-limited signal extrapolation: Models, discrete approximations, and noise. IEEE Transactions on Acoustics, Speech, and Signal Processing 31(6), 1492-1501 (1983). doi:10.1109/TASSP.1983.1164232

26. Zhou, X., Xia, X.: A sanz-huang conjecture on band-limited signal extrapolation with noise. IEEE Transactions on Acoustics, Speech, and Signal Processing 37(9), 1468-1472 (1989). doi:10.1109/29.31308

27. Zhou, X.-W., Xia, X.-G.: The extrapolation of high-dimensional band-limited functions. IEEE Transactions on Acoustics, Speech, and Signal Processing 37(10), 1576-1580 (1989). doi:10.1109/29.35396

28. Shi, J., Sha, X., Zhang, Q., Zhang, N.: Extrapolation of bandlimited signals in linear canonical transform domain. IEEE Transactions on Signal Processing 60(3), 1502-1508 (2012). doi:10.1109/TSP.2011.2176338

29. Adve, R.S., Sarkar, T.K., Rao, S.M., Miller, E.K.: The principle of analytic continuation-how to use it? In: Proceedings of ISSE'95 - International Symposium on Signals, Systems and Electronics, pp. 319-322 (1995). doi:10.1109/ISSSE.1995.497998

30. Zeng, G.L., Li, Y.: Fourier-domain analysis of the iterative landweber algorithm. IEEE Transactions on Radiation and Plasma Medical Sciences 1(6), 511-516 (2017). doi:10.1109/TRPMS.2017.2722863

Figure 1 Simplified generation modem of compressive OFDM signal. Figure 1:Attached in Figure/figure01.eps

Figure 2 Simplified recovery modem for compressive OFDM signals. Figure2: Attached in Figure/figure02.eps

Figure 3 BER of recovery for compressive OFDM signals with different compressed ratios and truncation filter modes. Figure 3:Attached in Figure/figure03.eps

Figure 4 NMSE of different iteration filter bandwidth Figure 4:Attached in Figure/figure04.eps

Figure 5 Regularized extrapolator for compressive OFDM signals. Figure 5:Attached in Figure/figure05.eps

Figure 6 NMSE of compressive OFDM with different compressed ratios. Figure 6:Attached in Figure/figure06.eps 
Figure 7 BER of compressive OFDM and SEFDM with different compressed ratios. Figure 7:Attached in Figure/figure07.eps

Figure 8 BER of compressive OFDM with different regularization parameters, OFDM and SEFDM. Figure 8:Attached in Figure/figure08.eps

Figure 9 ThroughputRate of compressive OFDM with different regularization parameters, OFDM and SEFDM. Figure 9:Attached in Figure/figure09.eps 
figure 01

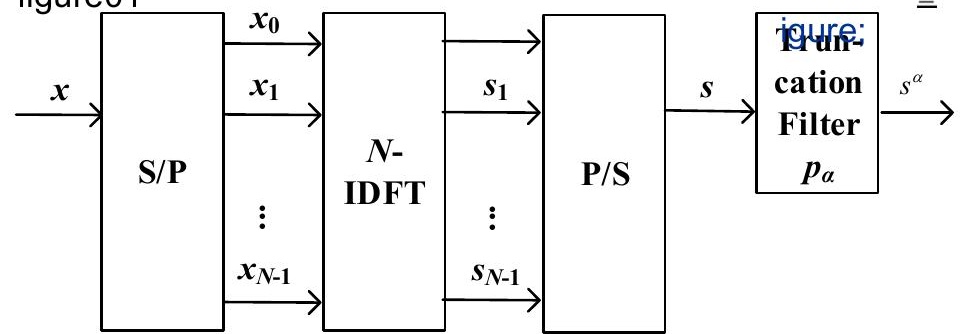




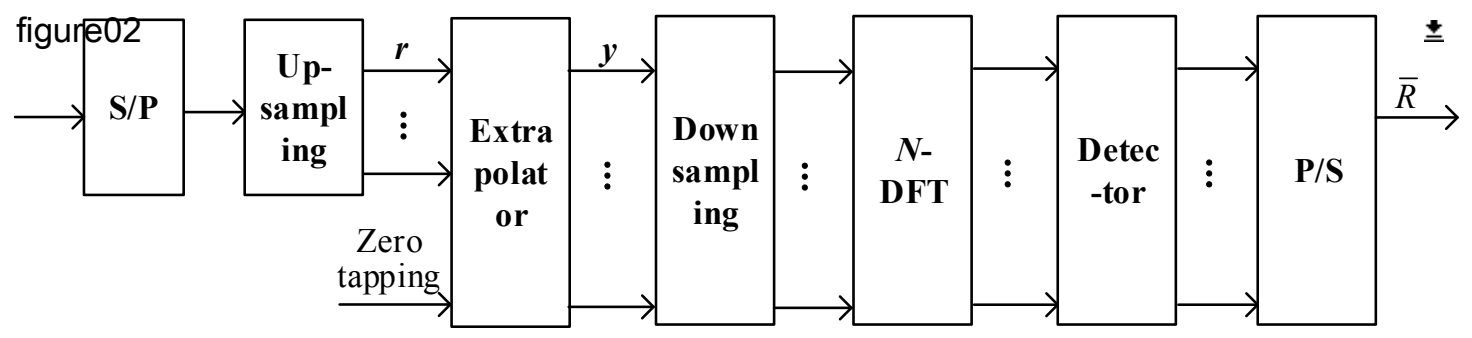




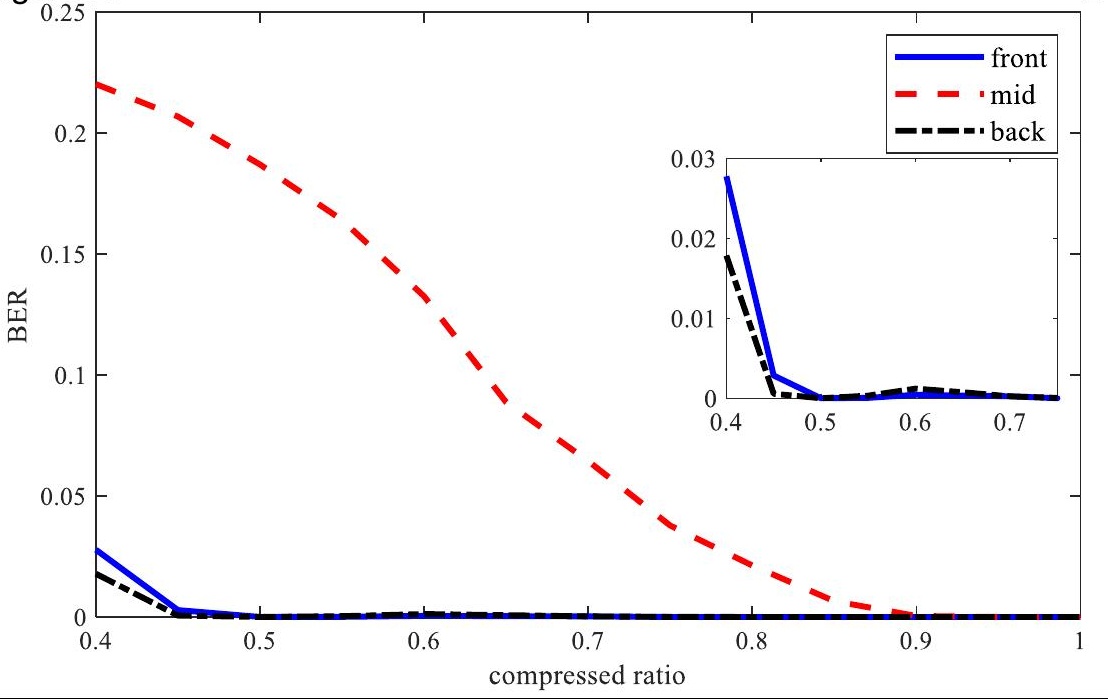




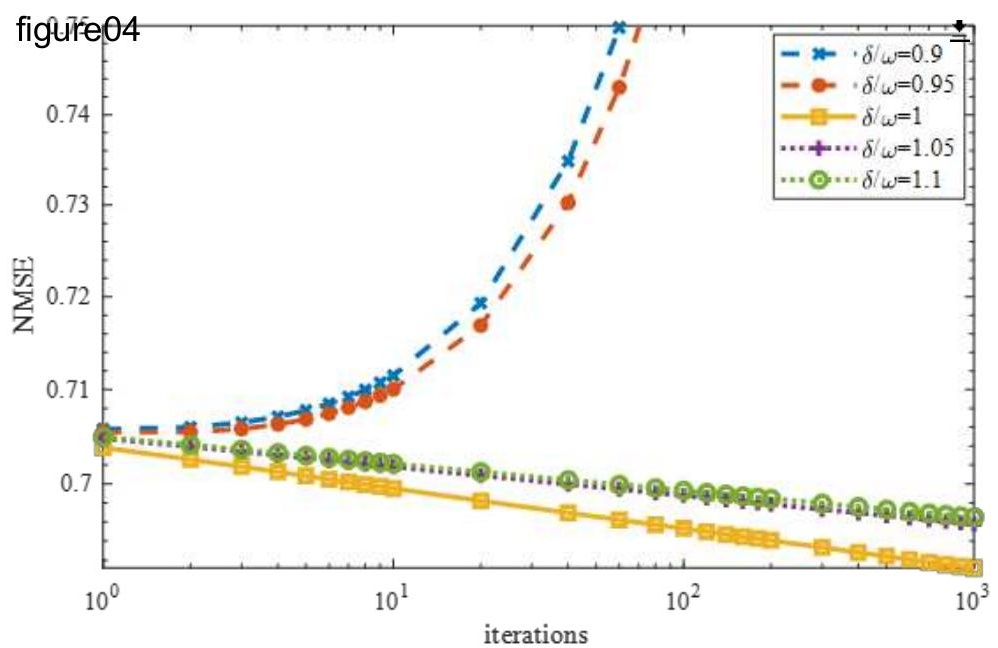


figure05

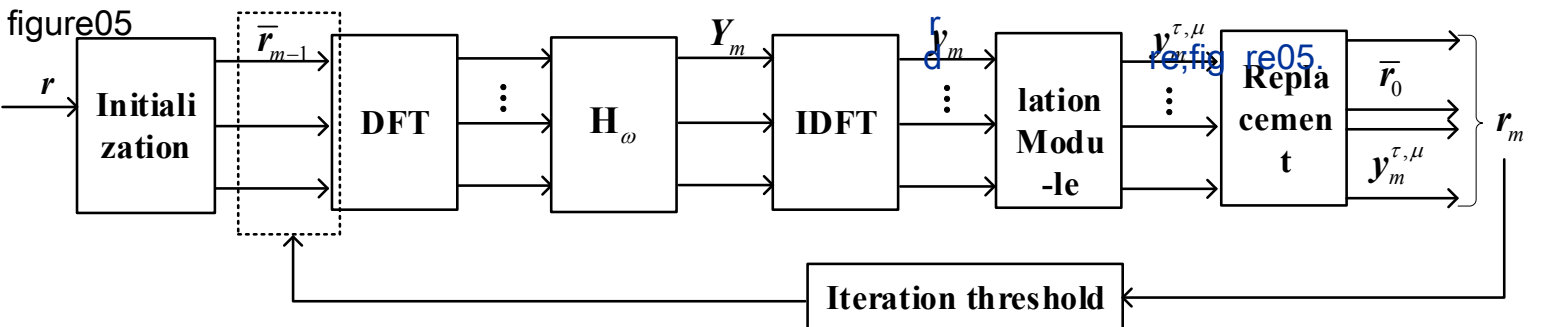




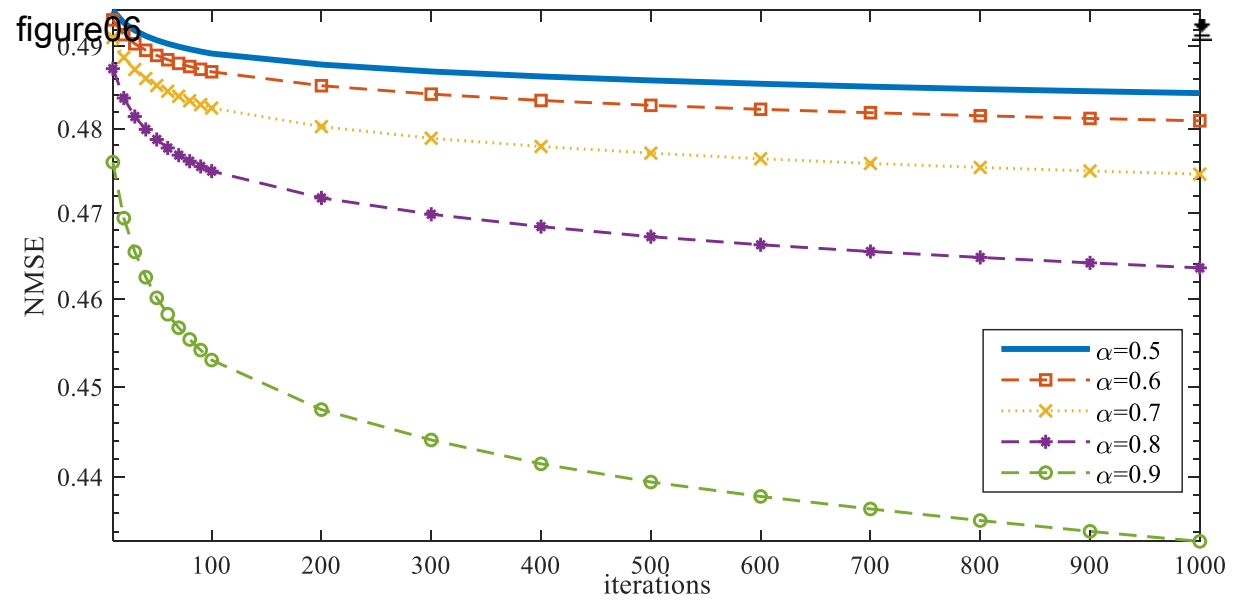




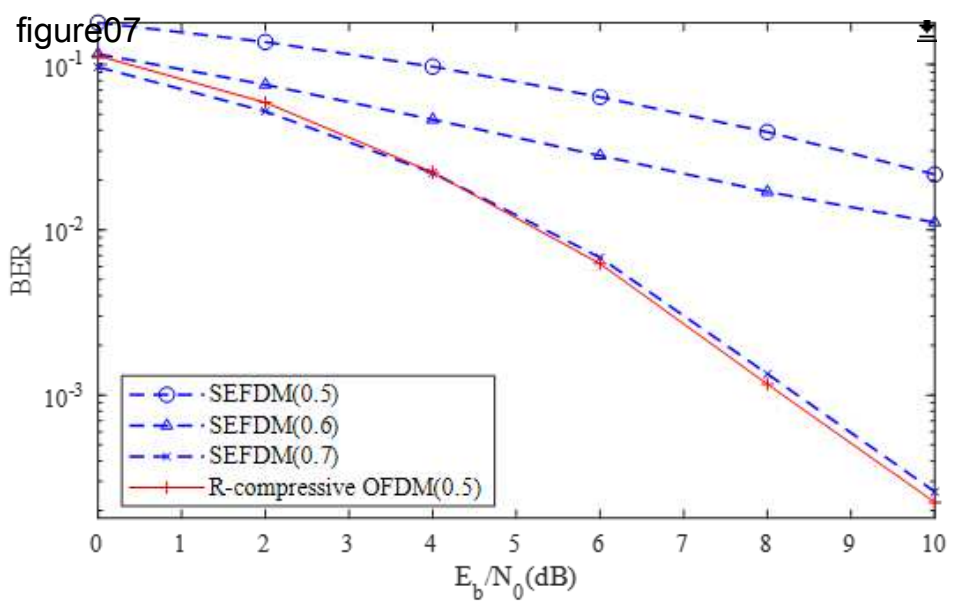




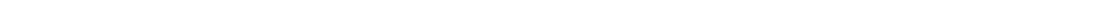

